\section{Profiling the Discordant Visceral Adipose Tissue-Ectopic Liver Fat Phenotype: Results from the Dallas Heart Study}

\author{
Authors: Cody McCoy, Colby Ayers, *Ian J. \\ Neeland \\ Department of Internal Medicine, University of Texas \\ Southwestern Medical Center, Dallas, Texas, USA \\ *Correspondence to ian.neeland@utsouthwestern.edu \\ Disclosure: Dr Neeland is a co-investigator on an \\ obesity trial funded by Novo Nordisk. The other \\ authors have declared no conflicts of interest.
}

Keywords: Cardiometabolic risk markers, liver fat, visceral fat.

Citation: EMJ Cardiol. 2020;DOI/10.33590 emjcardiol/2008313

\section{BACKGROUND}

The accumulation of visceral adipose tissue (VAT) and ectopic liver fat (ELF) generally parallel each other, but a proportion of individuals have discordant fat deposition. The cardiometabolic profile of individuals with a discordant phenotype is unknown.

\section{METHODS}

Amongst participants in the multiethnic Dallas Heart Study cohort, the authors examined the cross-sectional associations of VAT-ELF phenotypes with circulating biomarkers of insulin resistance, dyslipidaemia, inflammation, and subclinical atherosclerosis. VAT and ELF were assessed by MRI and magnetic resonance spectroscopy, respectively. Participants were stratified into phenotype groups: Iow VAT-Iow ELF, low VAT-high ELF, high VAT-low ELF, and high VAT-high ELF, defined by median sex and race-specific values for VAT and ELF. Associations between phenotype groups and biomarkers were adjusted for age, sex, race, menopausal status (females only), and BMI (referent low VAT-IOW ELF).

\section{RESULTS}

In multivariable models, high VAT-low ELF was significantly associated with insulin resistance (homeostatic model assessment of insulin resistance [HOMA-IR]); lower adiponectin concentration; higher low-density lipoprotein (LDL) cholesterol and more small, dense LDL particles; lower high-density lipoprotein (HDL) cholesterol and fewer large HDL particles; higher high-sensitivity C-reactive protein and myeloperoxidase levels; increased aortic wall thickness; and higher odds of hypertension, diabetes, and metabolic syndrome (Table 1). Low VAT-high ELF showed similar patterns, except that there was no independent association with aortic atherosclerosis, myeloperoxidase, or LDL cholesterol (Table 1).

\section{CONCLUSION}

High VAT-low ELF is associated with an adverse atherosclerotic, dyslipidaemic, dysmetabolic, and inflammatory phenotype, whereas the profile of low VAT-high ELF is more variable. These findings suggest phenotypic heterogeneity of abdominal and liver fat distribution with markers of cardiometabolic risk, and provide insight into the biological roles of VAT and liver fat in cardiometabolic disease. 
Table 1: Multivariable-adjusted linear and logistic regression models of relation of VAT-ELF phenotypes to biomarkers of cardiometabolic disease.

\begin{tabular}{|c|c|c|}
\hline & High VAT-Low ELF & Low VAT-High ELF \\
\hline Biomarker & B-coefficient or odds ratio $(95 \% \mathrm{Cl})$ & $\beta$-coefficient or odds ratio $(95 \% \mathrm{Cl})$ \\
\hline \multicolumn{3}{|l|}{ Insulin resistance } \\
\hline Glucose (mg/dL) & 0.020 & $0.040^{*}$ \\
\hline HOMA-IR (units) & $0.290^{*}$ & $0.320^{*}$ \\
\hline \multicolumn{3}{|l|}{ Adipokines } \\
\hline Adiponectin $(\mathrm{ng} / \mathrm{mL})$ & $-0.190 *$ & $-0.270^{*}$ \\
\hline \multicolumn{3}{|l|}{ Inflammation } \\
\hline $\mathrm{hs}-\mathrm{CRP}(\mathrm{mg} / \mathrm{dL})$ & $0.290^{*}$ & $0.270^{*}$ \\
\hline Myeloperoxidase $(\mathrm{ng} / \mathrm{mL})$ & $0.070^{*}$ & -0.003 \\
\hline \multicolumn{3}{|l|}{ Lipids } \\
\hline LDL-C (mg/dL) & $0.080^{*}$ & 0.040 \\
\hline LDL small & $0.360^{*}$ & $0.340^{*}$ \\
\hline Triglycerides (mg/dL) & $0.180^{*}$ & $0.290^{*}$ \\
\hline VLDL large & $0.320^{*}$ & $0.600^{*}$ \\
\hline $\mathrm{HDL}-\mathrm{C}(\mathrm{mg} / \mathrm{dL})$ & $-0.110^{*}$ & $-0.070^{*}$ \\
\hline HDL large & $-0.220^{*}$ & $-0.200^{*}$ \\
\hline \multicolumn{3}{|l|}{ Atherosclerosis } \\
\hline Aortic wall thickness (mm) & $0.060^{*}$ & 0.030 \\
\hline \multicolumn{3}{|l|}{ Risk Factors } \\
\hline Hypertension & $1.600(1.130,2.290)^{*}$ & $1.490(1.040,2.130)^{*}$ \\
\hline Metabolic syndrome & $3.470(2.410,5.000)^{*}$ & $2.880(2.010,4.140)^{*}$ \\
\hline Diabetes & $1.820(1.030,3.220)^{*}$ & $1.990(1.100,3.620)^{*}$ \\
\hline
\end{tabular}

Data presented are $\beta$-coefficients that represent the unit increase in the outcome for each phenotype group, or odds ratios that represent the odds of the outcome and associated 95\% confidence interval for each phenotype group of High VAT-LOW ELF or Low VAT-High ELF.

Model adjusted for age, sex, race, menopausal status (female only), BMI (referent low VAT-low ELF).

${ }^{*} \mathrm{p}<0.05$

Cl: confidence interval; ELF: ectopic liver fat; HOMA-IR: homeostasis model assessment of insulin resistance; LDL: low-density lipoprotein; LDL-C: low-density lipoprotein cholesterol; HDL: High-density lipoprotein; HDL-C: highdensity lipoprotein cholesterol; hs-CRP: high sensitivity C-reactive protein; VAT: visceral adipose tissue; VLDL: very low-density lipoprotein. 